
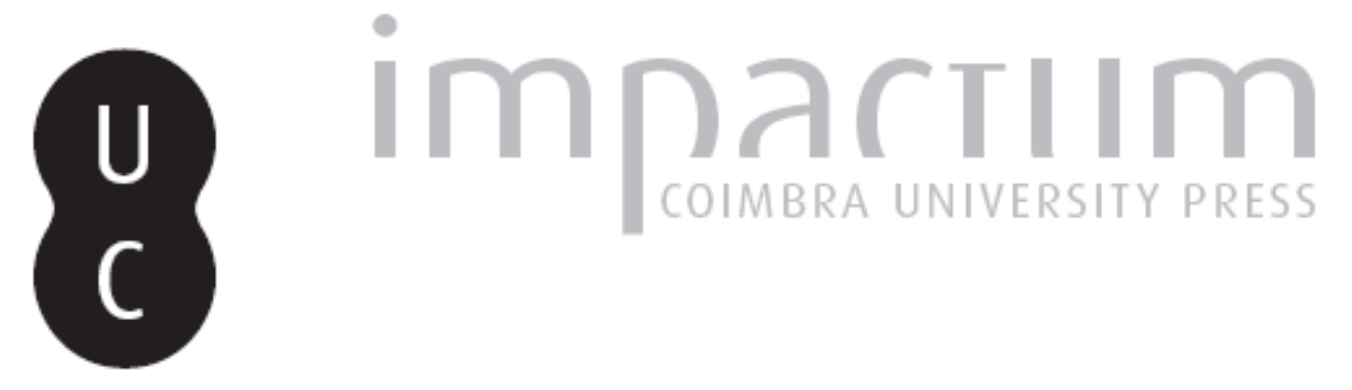

\title{
La monnaie et ses singularités
}

\section{Autor(es): Chaîneau, André}

Publicado por: Faculdade de Economia da Universidade de Coimbra

URL persistente:

URI:http://hdl.handle.net/10316.2/25241

DOI:

DOI:http://dx.doi.org/10.14195/2183-203X_37_6

Accessed : $\quad$ 26-Apr-2023 11:32:22

A navegação consulta e descarregamento dos títulos inseridos nas Bibliotecas Digitais UC Digitalis, UC Pombalina e UC Impactum, pressupõem a aceitação plena e sem reservas dos Termos e Condições de Uso destas Bibliotecas Digitais, disponíveis em https://digitalis.uc.pt/pt-pt/termos.

Conforme exposto nos referidos Termos e Condições de Uso, o descarregamento de títulos de acesso restrito requer uma licença válida de autorização devendo o utilizador aceder ao(s) documento(s) a partir de um endereço de IP da instituição detentora da supramencionada licença.

Ao utilizador é apenas permitido o descarregamento para uso pessoal, pelo que o emprego do(s) título(s) descarregado(s) para outro fim, designadamente comercial, carece de autorização do respetivo autor ou editor da obra.

Na medida em que todas as obras da UC Digitalis se encontram protegidas pelo Código do Direito de Autor e Direitos Conexos e demais legislação aplicável, toda a cópia, parcial ou total, deste documento, nos casos em que é legalmente admitida, deverá conter ou fazer-se acompanhar por este aviso.

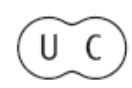




\section{N ○ TASEC O N O MICAS}

$\Delta$

\section{PAUL KRUGMAN'S TRIPLE DOCTORATE}

JORGE BRAGA DE MACEDO

PAUL KRUGMAN JOSÉ SILVA LOPES

ANDRÉ CHAîNEAU

ADÃO CARVALHO

ELSA DE MORAIS SARMENTO/

VANDA DORES /

GUIDA NOGUEIRA

ECONOMISTA MILITANTE

ECONOMICS IN THE CRISIS

COMMENT

LA MONNAIE ET SẸS SINGULARITÉS

FINANCIAMENTO PÚBLICO Ȧ I\&D EMPRESARIAL EM PORTUGAL

A COMPETITIVIDADE E A DIVERSIFICAC,ÃO DA FILEIRA FLORESTAL PORTUGUESA

$\Leftrightarrow$

$\Leftrightarrow$

$(-)$

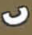

(2)

$\theta$

a

$\theta$ 37

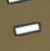
क

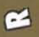
iv 


\section{4 (III) \& La Monnaie et ses Singularités}

André Chaîneau Professeur Emérite de l'Université de Poitiers

Qu'est-ce que la monnaie ? L'interrogation semble inutile, tout un chacun pensant connaître cet objet dont il se plaint généralement de manquer. Pourtant - et c'est bien là, la première singularité qu'on se plaira à souligner, les économistes semblent incapables d'en donner une définition simple et précise !

Cela commence avec John Richard Hicks qui, dans son célèbre article paru en 1935 dans la revue Economica " A Suggestion for simplifying the Theory of Money » classe les éléments de l'actif du bilan d'un agent économique selon leur degré de liquidité, de facilité à être transformé en monnaie. L'année suivante en 1936, John Maynard Keynes opére dans « la théorie générale de l'emploi, de l'intérêt et de la monnaie ", la substitution des mots. Le chapitre XVI débute par : " la préférence pour la liquidité ... est identique ... à la demande de monnaie ". II ne reste plus qu'à affirmer que la frontière entre monnaie parfaitement liquide et titres financiers n'est pas hermétique parce que certains titres émis à court terme, sans risques et liquidables à la demande peuvent être qualifiés de liquides (comme les dépôts sur livrets). Cette opinion est rapidement partagée par la plupart des auteurs. Hicks la réexpose magistralement dans ses « Critical Essays in Monetary Theory » en 1967.

Sur le plan conceptuel, on voit apparaître les termes de substitut monétaire, de quasi-monnaie. Sur le plan statistique, sont créés des agrégats gigognes avec l'agrégat monétaire M1 qui représente la masse monétaire au sens strict, M2 qui lui ajoute certains titres financiers et M3 d'autres encore un peu moins liquides. La thèse de l'extension de la masse monétaire qui se trouve ainsi validée puisque c'est M3 l'agrégat large qui est nommé masse monétaire ! Ce glissement s'est opéré sans réelle contestation. Les années 50 et 60 voient triompher le néokeynésianisme alors que le monétarisme n'avait pas bonne presse. La fourniture de plusieurs mesures pour un même concept ne paraissait et ne paraît toujours pas une absurdité mais, au contraire, une commodité pour les chercheurs !

On a au final réussi à noyer la monnaie liquide par nature dans un ensemble financier plus vaste et caractérisé par sa moindre liquidité. Sans aucun doute, il faut rappeler que la monnaie n'est que la monnaie. Mais encore faut-il pour cela, en énoncer toutes les singularités.

\section{Les singularités de l'actif monétaire}

\section{La monnaie est un actif original opposable fonctionnellement à tous les autres}

La monnaie est une forme d'avoir, un élément du patrimoine, disons un actif ; mais pas davantage qu'une maison, un véhicule ou un meuble. Comme pour tout autre actif (réel pour un bien, financier pour un titre), sa seule singularité réside dans la fonction qu'elle remplit, ou autrement dit dans l'utilité qu'on lui reconnaît. La monnaie est un moyen de paiement comme l'automobile est un moyen de transport. Au départ, l'actif monétaire n'est donc ni plus ni moins original qu'un quelconque actif non monétaire.

Mais à peine a-t-on affirmé sa banalité dans son essence, que l'actif monétaire se singularise immédiatement dans son existence. II s'oppose en effet à tous les actifs non monétaires pour deux raisons. D'abord, il sert à mesurer la valeur de marché de tous les autres actifs: il est leur dénominateur commun puisque tous ont un prix exprimé en unités monétaires. Ensuite la monnaie sert de contre-valeur lorsque ces actifs non monétaires font l'objet d'une transaction. Le vendeur qui livre un bien, un service ou un titre, exige une contrepartie de valeur équivalente : ce sera la quantité adéquate de monnaie que lui remet l'acheteur. La séparation est claire : d'un côté on a les actifs monétaires et de l'autre côté les actifs non monétaires que l'on vend pour de la monnaie ou que l'on achète contre de la monnaie. Cette opposition peut encore être formulée ainsi : d'une part il y a les actifs non monétaires-liquidables en monnaie (lors d'une transaction) et, d'autre part, la monnaie liquide par nature puisqu'elle circule d'acheteur à vendeur à chaque transaction successive. 


\section{La monnaie est un actif créé par les banques ex nihilo, sans coût de production}

La monnaie s'est dématérialisée au cours du temps. De nos jours, elle est une monnaie-signe, sans valeur intrinsèque, sans coût de production. Ainsi, l'émission de monnaie manuelle (les billets) ne supporte que les frais de leur impression ; mieux encore, l'émission de monnaie scripturale (les dépôts monétaires qui circulent par chèques, virements, cartes bancaires...) n'exige qu'une simple écriture de crédit au compte d'un agent vendeur de devises ou emprunteur bancaire. Elle est pourtant l'équivalent-valeur de tout ce qui est négociable : biens, services et titres. C'est une nouvelle singularité que cette gratuité de l'émission de monnaie. La monnaie qui possède un pouvoir d'achat égal à sa valeur nominale, ne coûte rien à produire! Logiquement elle ne devrait rien valoir ! D'où lui vient cette dimension d'actif ? Elle résulte d'un consensus social qui, par exemple, fait accepter un billet pour 100 ou 50 euros si l'une de ces deux valeurs est simplement imprimée dessus. Mais alors les producteurs de monnaie, ses émetteurs détiennent un pouvoir fabuleux, celui de pouvoir créer des moyens de paiement pour régler leurs propres transactions ! Cette commodité extraordinaire est bien sûr limitée. D'abord seules les banques ont cette faculté et, ensuite, elles ne peuvent y recourir que lorsqu'elles achètent des devises (créances sur l'extérieur) ou encore des titres représentatifs de crédit (crédits à l'économie ou à l'Etat) et, enfin, cette activité est contrôlée par la politique monétaire.

Imaginons qu'une banque accorde un crédit à une entreprise $\mathrm{E}$. Elle achète un titre (de créance pour elle puisqu'elle est le prêteur) qui figure naturellement à son actif ; et elle paie cette acquisition simplement en créditant le compte qui enregistre les dépôts monétaires de son client $E$ et qui figure à son passif. Bref, cet achat ne lui coûte que la peine de passer une écriture. On dit qu'il y a eu monétisation du titre parce qu'il y a eu une création de monnaie égale à la valeur nominale du titre acheté, c'est-à-dire du prêt accordé. En effet, cette opération n'a réduit les encaisses monétaires d'aucun agent économique mais par contre a augmenté celles de l'emprunteur.

Le caractère ex nihilo (approximativement) de la création monétaire a deux conséquences. La première est que la production de monnaie se fait sans coût important (encore ne faut-il pas négliger les coûts de gestion de cette monnaie qui sont supportés par les banques). La seconde est que la banque a prêté sans avoir eu à emprunter : elle n'a de relation qu'avec le client auquel elle prête. Dans le raisonnement qui vient d'être conduit, la banque est réduite à n'être qu'un organisme créateur de monnaie. Elle l'est et c'est son privilège. Mais elle peut aussi comme les autres agents économiques prêter ce qu'elle a emprunté ou ce qu'elle a épargné sur son revenu d'exploitation. Mais comme cette étude concerne la monnaie, le mot banque désigne ici l'institution créatrice de monnaie.

La conclusion est que les banques disposent seules, au passif de leur bilan, d'un type particulier de ressources : celui de la création monétaire. Les autres agents non bancaires ne recensent au passif de leur bilan, que leurs capitaux propres (épargnés par eux-mêmes) et leurs endettements (empruntés par eux). Seules les banques peuvent ajouter à ces ressources classiques, celle de l'émission monétaire.

\section{A l'actif monétaire ne correspond pas une dette bancaire}

L'idée d'une monnaie dette bancaire est souvent défendue. Pourtant, elle ne tient pas. Pour son émetteur, la monnaie n'est pas une dette comme l'est un titre financier qui est émis lors d'une opération prêt-emprunt, qui est remboursable et rémunéré par un intérêt. Sans aucun doute, la monnaie émise se retrouve au passif de la banque et à l'actif de l'agent qui la détient ; de même le titre émis par l'emprunteur figure à son passif et se retrouve à l'actif du prêteur qui le possède. Cette similitude comptable explique le succès de l'idée de monnaie-dette. Mais on peut établir que si le titre d'emprunt représente bien une dette, il n'en va pas de même pour la monnaie émise. En effet, sa fonction de moyen de paiement la contraint à circuler indéfiniment lors des transactions, dans la communauté du secteur non bancaire où elle se trouve en quelque sorte piégée. C'est ce rôle d'intermédiaire des échanges qui exclut toute idée de remboursement, d'un remboursement 
au demeurant incompréhensible. Avec quoi pourrait-on rembourser la monnaie qui a été déclarée inconvertible, si ce n'est avec une autre forme monétaire ? Le résultat est que les banques émettrices de monnaie disposent de ressources qui, quoique étant très volatiles (elles bougent tout le temps entre leurs utilisateurs) sont globalement parfaitement stables : elles ne sortent jamais du bilan des banques, ces dernières étant indifférentes à l'identité de ses détenteurs. Aussi l'idée d'assimiler la monnaie émise par les banques à une dette bancaire est dénuée de sens.

\section{La monnaie est irréductible à un titre financier}

II faut insister sur ce point parce que la monnaie et les titres financiers liquidables à la demande sans risque paraissent substituables. Par exemple, un ménage peut considérer que détenir une encaisse monétaire oisive équivaut à posséder des dépôts sur un livret d'épargne s'il est indifférent à l'intérêt versé ou à l'obligation de se déplacer pour récupérer son argent dans le cas de l'épargne placée.

Or la monnaie est le seul actif liquide. Elle circule naturellement lors des transactions. Cette propriété établit sa nature liquide. Aucun autre actif ne peut la concurrencer sur ce terrain. Les autres actifs non monétaires ne sont que liquidables sur un marché : ils peuvent être vendus et transformés en monnaie. La simple considération du vocabulaire liquide - liquidable suffit à établir la spécificité de la monnaie seule intrinsèquement liquide.

Pourtant, beaucoup d'économistes veulent que l'on confonde la monnaie actif liquide et divers actifs financiers liquidables soit à vue soit à très court terme, à leur prix d'émission et en conséquence sans risque. Pour justifier cette assimilation, ils constatent en premier lieu que la monnaie est une réserve de valeur nominale constante et liquide et en second lieu que dans le patrimoine des agents économiques, la monnaie thésaurisée est concurrencée, mieux remplacée par divers titres financiers courts dont les dépôts sur livret. Il est indiscutable que posséder un dépôt disponible à la demande et rémunéré sur un livret est préférable à détenir une encaisse monétaire oisive elle aussi disponible à la demande mais non rémunérée par un intérêt.

Peut-on déduire de ce fait que les dépôts sur livret sont l'équivalent de la monnaie ? La réponse est négative. Ils ne sont substituables qu'à la seule monnaie thésaurisée, à la seule partie de l'encaisse monétaire qui oisive, au repos, ne remplit pas sa fonction de moyen de paiement parce qu'elle est en attente d'utilisation. La masse monétaire, la quantité de monnaie qui existe dans une économie, se partage entre la monnaie active qui circule entre acheteurs et vendeurs et la monnaie oisive qui n'est pas utilisée dans les échanges. Cette dernière représente l'équivalent d'un placement en monnaie non rémunéré. Qu'il soit concurrencé par les placements financiers eux rémunérés, qu'il le soit encore plus par les placements financiers liquidables instantanément, n'est pas une constatation bien troublante. Tout au plus peut-on affirmer que la monnaie est essentiellement un actif de paiement et très accessoirement (de façon négligeable de nos jours) un actif de placement. Mais en aucun cas, on ne peut en déduire en sens inverse que certains actifs financiers sont quasiment de la monnaie.

A ce stade du raisonnement, ces économistes partisans d'un agrégat liquidités plus large que l'agrégat monétaire, affirment que « liquidable facilement, sans coût et sans risque » d'une part et « liquide » d'autre part, sont pratiquement synonymes sur un plan pratique. La réponse est négative deux fois. D'abord, si chaque titre liquidable à la demande peut être liquidé individuellement, l'ensemble de ces titres ne l'est jamais parce qu'ils forment un ensemble en voie de croissance : les souscriptions nouvelles l'emportent régulièrement sur les remboursements. Le résultat est que ces titres ne sont que potentiellement liquidables et jamais liquidés dans les faits. Ainsi globalement ils représentent non pas de la monnaie mais une épargne stable. Ensuite l'existence de ces titres a renforcé la singularité de l'actif monétaire. L'explication est simple. En concurrençant victorieusement la monnaie oisive, ils ont réduit la masse monétaire à la seule monnaie active, celle qui remplit la fonction de moyen de paiement. L'épargne liquidable à la demande ou à court terme, au lieu de réaliser la confusion du concept de monnaie, a au contraire accentué son originalité. 
Par ailleurs, les création et destruction de monnaie ne peuvent pas être confondues avec les création et destruction de titres. La production des titres au moment de la réalisation de l'opération prêt - emprunt a des conséquences totalement différentes selon que le prêteur est un agent non bancaire ou une banque. II en va de même lors de la destruction du titre qui s'opère au moment du remboursement.

Le premier cas est celui où le prêteur ultime est un agent non bancaire, par exemple, un ménage. Celui-ci a épargné un certain pouvoir d'achat, une certaine quantité de monnaie. II peut la prêter directement à une entreprise ou à l'Etat en leur achetant un titre de créance pour lui et d'endettement pour eux. Mais il peut aussi la leur prêter de façon indirecte en prêtant d'abord à une institution financière qui lui vendra un titre sur elle-même et ainsi collecte son épargne, épargne que cette institution financière prêtera par la suite à l'entreprise ou à l'Etat en leur achetant un titre financier. Le commentaire est évident : dans tous les cas, le ménage d'abord épargne, c'est-à-dire gèle un certain pouvoir d'achat qu'il transfère ensuite à un emprunteur qui s'en servira à sa place. Au terme de cette opération de prêt-emprunt, c'est l'inverse : l'emprunteur met de côté le pouvoir d'achat nécessaire au remboursement pour le remettre à son prêteur. Ainsi il n'y a que la circulation d'un pouvoir d'achat préexistant du prêteur vers l'emprunteur au départ, puis avec l'arrivée du terme, sa restitution par l'emprunteur au prêteur. On ne constate ni création, ni destruction de pouvoir d'achat monétaire.

Mais - et c'est le second cas - lorsque le prêteur est une banque, celle-ci « monétise » le titre d'endettement vendu par l'emprunteur. Cette expression ne signifie pas qu'elle le transforme en monnaie. Mais elle traduit le pouvoir extraordinaire des banques qui peuvent créer de la monnaie pour payer leurs acquisitions. Ainsi lorsque le système bancaire SB accorde 100 de crédits à un ensemble d'emprunteurs EMP, il se contente de créditer de 100 les comptes bancaires monétaires de ces agents EMP. L'opération se représente ainsi :

ACTIF Prêts nets aux emprunteurs EMP + $100 \longrightarrow$ PASSIF Dépôts monétaires de EMP +100

Les emprunteurs EMP disposent maintenant d'un pouvoir d'achat additionnel qui n'existait pas avant l'opération prêt-emprunt. Et l'on peut comprendre qu'une monétisation trop importante de crédits par les banques conduit nécessairement à l'inflation si elle ne trouve pas plus de biens à échanger sur les marchés. Inversement, le remboursement des crédits aux banques conduit à leur démonétisation, à une destruction de monnaie. Le compte bancaire de l'emprunteur est débité, réduit du montant de sa dette ; ce qui, toutes choses égales par ailleurs, diminue la quantité de monnaie disponible dans l'économie.

La conséquence est que la distinction entre un prêteur non bancaire qui offre des ressources d'épargne (les siennes ou celles qu'il a collectées) et un prêteur bancaire qui peut créer ses fonds prêtables, est totale. Les banques peuvent être responsables d'une inflation (trop de monnaie) ou d'une déflation (pas assez de monnaie), mais pas les prêteurs du secteur non bancaire, secteur qui contient aussi les institutions financières qui exercent la fonction de collecte d'épargne. Le fait que les banques cumulent le pouvoir de collecter l'épargne et le pouvoir de créer la monnaie n'a aucune importance logique puisque c'est leur pouvoir exclusif de création monétaire qui les distingue de tous les autres agents économiques.

En résumé, la monnaie est créée ex nihilo par les banques qui possèdent le privilège formidable de produire de la monnaie sans supporter de coûts de production : elles monétisent les devises qu'elles achètent et les crédits qu'elles accordent, c'est-à-dire financent ces emplois en créant la monnaie correspondante. La célèbre formule « les prêts font les dépôts » est un excellent résumé de ce pouvoir. La logique comptable naturelle, générale qui veut que l'on emploie ses ressources est ici exceptionnellement inversée au profit des banques : ce sont en effet les emplois qui vont engendrer les ressources correspondantes ! 2- Dans cette activité de monétisation, les banques ne sont pas obligées d'emprunter quoi que ce soit. La monnaie qui figure comptablement à leur passif, ne peut pas être une dette bancaire, d'une part parce que sa 
fonction de moyen de paiement assure sa pérennité (la masse monétaire dont chaque élément est très volatil, est cependant globalement parfaitement stable et même en voie de croissance) et d'autre part parce qu'il est difficile d'imaginer avec quoi la monnaie pourrait être remboursée. 3- La création de monnaie est nécessairement la création d'un pouvoir d'achat nouveau potentiellement inflationniste alors que la création de titres même courts (c'est-à-dire à très brève échéance) n'assure que la circulation d'un pouvoir d'achat préexistant et donc n'est pas potentiellement inflationniste.

\section{Les singularités du couple offre - demande de monnaie}

\section{L'offre de monnaie précède la demande de monnaie !}

Le point d'exclamation s'impose parce que pour tous les biens, tous les services, tous les titres, c'est la relation inverse qui prévaut. II n'y a offre que s'il y a eu demande. L'offreur peut essayer de créer sa propre demande par la publicité, mais s'il ne réussit pas, il n'offrira pas. Alors pourquoi l'actif monétaire échappe-t-il à cette loi générale ?

L'explication est simple. La monnaie est offerte à qui ne la demande pas ! L'emprunteur ne veut pas la monnaie qu'il sollicite ; il veut la ressource - l'endettement bancaire - qui lui permettra d'acquérir le bien désiré : un équipement pour une entreprise, un logement pour un ménage etc. Sans doute reçoit-il de la monnaie, mais à peine l'a-t-il qu'il s'en débarrasse :

Actif : Monnaie Passif : Endettement bancaire

Actif : Bien désiré acquis Passif : Endettement

puis quasi - instantanément

La conséquence est que cette monnaie va commencer un processus de circulation qui la fera détenir par la communauté des agents économiques qui pourtant n'a pas pris part à sa création, à son offre.

En résumé la monnaie est offerte à des agents emprunteurs des banques, qui ne leur demandent pas de la monnaie mais des crédits.

\section{La demande de monnaie s'adapte à l'offre !}

Cette seconde singularité résulte logiquement de celle qui précède mais apparaît peut-être encore plus extraordinaire.

Supposons une surproduction de monnaie. Les banques et leurs emprunteurs se mettent d'accord sur un montant de crédits qui, une fois monétisés, conduisent à une création monétaire excessive parce qu'elle dépasse les besoins instantanés de monnaie pour les échanges. Les agents économiques ont conscience de cet excès de liquidité. Bien évidemment ils ne détruisent pas ce « trop » de monnaie mais le dépensent sur les divers marchés. La demande supplémentaire financée par cette surabondance de monnaie peut 1 - conduire à une expansion de l'offre (effet-quantité heureux) mais plus sûrement 2- conduire à l'inflation des prix (effet-prix malheureux). Ce que l'on constate est qu'au terme de ces deux effets, le besoin de monnaie pour les transactions a augmenté et qu'à la fin de ce processus d'ajustement, la demande a rattrapé l'offre et la « surliquidité » initiale génératrice du déséquilibre a disparu. Ainsi la demande s'est ajustée à l'offre parce que le besoin a augmenté avec la hausse des prix ou encore l'excès initial de liquidité a disparu parce que le pouvoir d'achat de chaque signe monétaire (et donc son utilité) s'est auto-réduite avec cette inflation des prix.

Le cas inverse d'une sous-production monétaire est exceptionnel. Mais théoriquement son analyse serait la même. Une situation de rareté monétaire, d'illiquidité, conduit les agents économiques à restreindre leurs transactions. En conséquence, leurs besoins d'encaisses monétaires se réduisent, et ainsi au terme d'un processus de contraction, la demande de 
monnaie redevient égale à une offre qui au départ était pourtant insuffisante. La seule chose à noter est une asymétrie avec la situation précédente. Les prix étant rigides à la baisse, ce sont les quantités qui se contractent : on comprend alors sans peine que le spectre de la déflation est souvent évoqué même si cette déflation est en fait toujours évitée depuis la grande crise de 1929.

La monnaie se révèle être encore à ce titre un actif original. Aucun autre ne possède le pouvoir d'auto-détruire son utilité pour être plus demandé s'il est trop offert (inflation) ou d'auto-créer son utilité pour être moins demandé s'il n'est pas assez offert (déflation).

\section{Les singularités des flux monétaires du circuit économique}

\section{Les trois flux monétaires fondamentaux du circuit économique : un pour le faire fonctionner et deux pour le perturber.}

L'activité économique d'un pays au cours d'une période peut être représentée par la somme des échanges monétaires qui réunissent les agents économiques. La nécessité de ces transactions est évidente. Pour acheter, tout un chacun doit vendre. Sur un plan réel, il faut offrir pour pouvoir demander. Ainsi la vie économique saisie sous sa dimension monétaire s'explique par cette contrainte des agents économiques : offrir-vendre pour demander-acheter. Mais comme des milliards de transactions sont passées entre des dizaines de millions d'opérateurs, il est nécessaire de simplifier la réalité. Cette schématisation est permise parce que l'on peut opposer deux pôles d'agents - les ménages et les entreprises - qui adoptent deux comportements contraires d'offres et de demandes sur deux ensembles de biens et services distincts - les facteurs de production et les biens produits -. On obtient alors le circuit économique le plus simple : 1- les ménages propriétaires des facteurs de production (travail, ressources naturelles, biens de production eux-mêmes ou services rendus par ces biens) les vendent aux entreprises qui les achètent pour pouvoir produire et qui leur versent leurs revenus $Y$ (qui comprend les salaires, les loyers, les profits distribués etc) ; 2- les ménages munis de ces ressources monétaires achètent alors aux entreprises, pour un montant $D$, les biens de consommation que celles-ci ont produits et leur vendent.

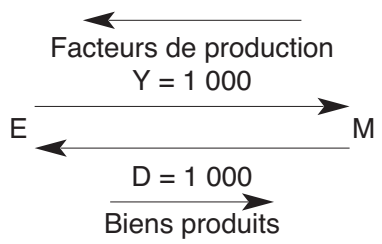

Cette représentation, la plus simple du circuit, réduite aux flux monétaires $Y$ et $D$, suffit pour faire quatre remarques. La première est que derrière $Y$ et $D$, il y a une quantité de monnaie égale à 1000 qui circule de $E$ à $M$ puis de $M$ à $E$ et que l'on peut qualifier d'active parce qu'elle remplit sa fonction de moyen de paiement. La deuxième est que l'existence de ces 1000 de monnaie n'est pas expliquée. D'où viennent-ils ? Nul ne le sait. Ils sont là. Alors on explique qu'il s'agit d'une variable exogène, c'est-à-dire d'une variable qu'on n'explique pas ! Ce que l'on peut écrire est que ce circuit économique fonctionne à périmètre monétaire constant ou encore qu'il correspond à un circuit monétaire fermé. La troisième remarque est que l'égalité $D=Y$ assure le bouclage du circuit économique : les ménages ont les revenus $Y$ de leurs dépenses $D$ et les entreprises les revenus $D$ de leurs dépenses $Y$. Cela garantit que les entreprises pourront à nouveau verser 1000 de revenus aux ménages et que le circuit pourra repartir pour un nouveau tour. Cette égalité $\mathrm{D}=\mathrm{Y}$ est la condition monétaire de la pérennité de ce circuit. La quatrième est que tous les agents économiques de ce circuit $\mathrm{M}$ et $\mathrm{E}$ sont soumis à la contrainte de leur équilibre budgétaire : ils ne peuvent dépenser plus que leurs recettes. Cette constatation est souvent négligée parce que l'on présuppose qu'il en va ainsi pour tous les agents économiques. C'est une erreur. Le secteur bancaire SB doté du pouvoir de monétisation fait exception pour le 
montant de son émission monétaire, et non seulement, il n'est pas implicitement inclus dans ce schéma de circuit mais surtout il ne peut pas y entrer. C'est un point très important. Toutes les représentations du circuit économique qui « oublient » le secteur bancaire en tant que créateur de monnaie, sont des représentations amputées.

II faut donc le prendre explicitement en considération, en supposant qu'il accorde 50 de crédits monétisés aux entreprises.

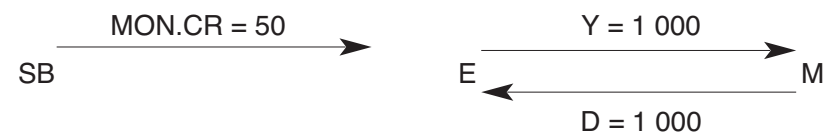

Les conséquences sont les suivantes ; 1- la quantité de monnaie augmente de 50, 2- les ressources des entreprises qui sont la somme des deux flux D + MON.CR, passent à 1050 , et 3- comme les entreprises ont emprunté 50 pour dépenser, elles achèteront davantage de facteurs de production aux ménages soit 1050 au lieu de 1000 comme auparavant. La création monétaire des banques constitue un flux d'injection monétaire qui dilate le circuit.

II faut maintenant reconnaître également aux ménages, le pouvoir monétaire de modifier la quantité de monnaie active dans les échanges. Ils l'exercent en thésaurisant une partie de leurs revenus monétaires, c'est-à-dire en ne la dépensant pas. Imaginons qu'à partir de la situation initiale, ils thésaurisent 50 de monnaie qu'ils conservent oisive dans leurs encaisses.

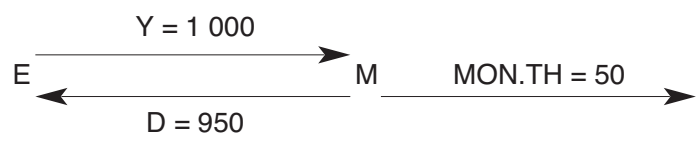

Les conséquences sont les suivantes : 1- les dépenses des ménages tombent à 950, 2 - la quantité de monnaie active également, 3- les entreprises n'ayant plus que 950 de recettes n'achèteront des facteurs de production que pour ce montant, 4- en conséquence le circuit économique se contracte.

Evidemment les ménages peuvent déthésauriser la monnaie oisive qu'ils détiennent déjà. Alors le flux s'inverse et représente une injection monétaire.

En résumé, le circuit économique fonctionne parce qu'il existe une quantité de monnaie active (un stock) qui circule entre acheteurs et vendeurs à l'occasion des transactions. II se contracte parce que la thésaurisation des ménages représente une fuite hors du circuit. II se dilate parce qu'un flux de monnaie, soit créée par le secteur bancaire soit déthésaurisée par les ménages, représente une injection dans ce circuit. Le circuit économique saisi dans sa dimension monétaire peut être comparé à un circuit hydraulique doté de deux robinets, l'un de sortie de l'eau et l'autre d'alimentation en eau ; il serait difficile de nier l'importance des flux qui passent à travers ces deux robinets. On établit, là encore, une grande singularité des flux monétaires.

\section{L'importance de l'injection monétaire nette dans le circuit économique}

La quantité de monnaie active augmente avec la création monétaire MON.CR et diminue avec sa thésaurisation MON.TH. Sa variation est donc donnée par la différence : MON.CR MON.TH.

Que se passe-t-il si cette injection monétaire nette est nulle ? La quantité de monnaie active ne change pas. Le circuit peut continuer à fonctionner avec les mêmes valeurs de revenu global et de dépense globale ; sa pérennité est assurée sur le plan monétaire. Mais nos économies connaissent une croissance économique réelle. Le volume des échanges augmente régulièrement 
et comme le niveau général des prix ne baisse pas, le besoin de monnaie active supplémentaire se fait ressentir sans cesse. Une constante injection monétaire nette de monnaie se trouve de ce fait légitimée. Si elle ne se réalisait pas, les échanges seraient freinés. Avec une relation de proportionnalité entre les échanges et la quantité de monnaie active, on peut déduire que le taux de croissance de cette monnaie doit être égal au taux de croissance réelle de l'économie.

Mais que se passe-t-il s'il lui est supérieur ? Il y a maintenant abondance relative de monnaie par rapport aux biens services et titres échangeables. II en résulte une inflation des prix et une destruction du pouvoir d'achat de chaque signe monétaire qui justifient un besoin accru de monnaie pour les transactions. Lorsque ce processus inflationniste est achevé, l'excès initial de monnaie a disparu. Pour simplifier, il y avait $10 \%$ de monnaie en trop ; les prix ont augmenté de $10 \%$ et les besoins de monnaie également ; il n'y a plus « trop » de monnaie. Le problème est que ce rééquilibrage par l'inflation ne se fait pas aussi simplement. D'abord tous les prix ne montent pas de $10 \%$ mais seulement leur moyenne : le fait que certains prix s'envolent et que d'autres stagnent, est très déstabilisant pour I 'économie. Ensuite cette inflation provoque des dommages collatéraux : spéculation déraisonnable sur des biens considérés comme des valeurs-refuges, dépréciation des titres financiers du type obligataire avec appauvrissement des prêteurs, hausse des taux d'intérêt, dépréciation de la monnaie sur les marchés de change, raccourcissement de l'horizon économique des agents qui préfèrent le présent au futur, enrichissement par plus-values et non par participation à l'activité économique. etc. Pour faire court, la hausse des prix éponge l'excès de monnaie - ce qui est un bien - mais en laissant s'installer une « économie de casino » - ce qui est un mal -.

La conclusion est que l'injection monétaire nette doit être telle que, d'une part, elle ne bloque pas la croissance économique en étant trop faible et que, d'autre part, elle n'engendre pas l'inflation en étant trop abondante.

\section{La gestion de l'approvisionnement monétaire d'une économie}

La politique monétaire qui contrôle l'approvisionnement de l'économie en monnaie, possède une première singularité. Dans une économie de marchés totalement libres, elle se donne un objectif de rationnement monétaire. Elle veut que le circuit économique dispose d'une quantité de monnaie active suffisante pour une facile réalisation de ses échanges. La norme retenue est légèrement biaisée du côté de l'inflation parce que l'on ne veut pas courir le risque d'une déflation. Elle est : taux de croissance monétaire = taux de croissance réelle + taux d'inflation jugé socialement tolérable (généralement $2 \%$ ). Cette intrusion d'un contrôle quantitatif dans une économie de marchés est quelque peu surprenante.

Une seconde singularité est à prendre en considération. Cette économie totalement décentralisée, ignorant toute forme de planification, doit sur le plan monétaire, être dirigée autoritairement du centre, par une administration. Une économie de marchés libres pour tous les biens, services et titres, est simultanément une économie administrée sur le plan monétaire. Pour mettre en place ce rationnement, il faut une autorité centrale qui s'impose à tous. L'administration qui en a la charge, est la Banque Centrale. C'est elle qui est la responsable finale de l'activité de création monétaire des banques. Cette dimension réglementaire du contrôle monétaire d'une économie apparaît tellement nécessaire que des économistes pourtant très libéraux ont même imaginé d'aller plus loin en remplaçant le pouvoir discrétionnaire de la Banque Centrale qui peut faire évoluer sa politique, par une règle légale qui définirait une norme intangible de croissance de la masse monétaire.

Tout ceci est surprenant à première vue, mais s'explique simplement. II suffit de compendre qu'une économie même absolument libérale a besoin pour fonctionner d'une monnaie qui est un bien atypique : un bien appropriable individuellement mais à usage collectif. Ce bien possède donc une nature tout à fait originale et en conséquence différente de tous les autres actifs échangeables. 
En conclusion, la monnaie est la monnaie et n'est que la monnaie. Elle possède trop de singularités.

1 - Elle représente un pouvoir d'achat quoiqu'elle soit une création ex nihilo.

2 - Désirée par tout un chacun, elle est demandée par qui n'en veut pas ! Son offre précède sa demande !

3 - Ceux qui ne l'ont pas demandée, sont obligés de la détenir ! Sa demande suit son offre !

4 - Le plus souvent surproduite, son montant réel est pourtant toujours optimal parce qu'elle peut auto-détruire son utilité ! Sa demande s'ajuste à son offre !

5 - L'actif monétaire fait l'objet d'une double demande à la fois simultanée et contradictoire ! D'abord une demande de monnaie active destinée à être dépensée et ensuite une demande de monnaie oisive destinée à ne pas être dépensée ! Aussi son rôle dans les transactions est celui de la monnaie active égale à la masse monétaire moins le montant qui en est thésaurisé.

6 - Le circuit économique est soumis à une double influence monétaire : celle de la création monétaire courante qui le dilate et celle de la thésaurisation courante qui le contracte. Le solde, l'injection monétaire nette, quasiment toujours positif entraîne la croissance de la valeur des flux du circuit économique, croissance qui se partage entre croissance réelle en volume si celle-ci est possible, et croissance nominale des prix c'est-à-dire inflation.

7 - La représentation minimale du circuit économique ne peut se limiter au couple Ménages Entreprises. Elle doit prendre en considération le Secteur Bancaire dans sa fonction de création monétaire.

8 - Pour éviter l'inflation qui génère de grands désordres économiques, la production de monnaie est soumise à un contrôle quantitatif discrétionnaire des autorités monétaires. Ainsi une économie de marchés libres est soumise à une tutelle administrative qui se justifie parce que la monnaie bien appropriable individuellement est simultanément un bien à usage collectif. 